\title{
Characteristics of circulating endothelial cells obtained from non-ST-segment elevation myocardial infarction patients with additional diastolic dysfunction of left ventricle observed in echocardiography
}

\author{
Pawel Burchardt ${ }^{1,2,3}$, Maura Farinacci ${ }^{4}$, Magdalena Mayer ${ }^{5}$, \\ Klaus Luecke ${ }^{6}$, Thomas Krahn ${ }^{7}$ Jaroslaw Manczak ${ }^{2}$, \\ Marek Slomczynski ${ }^{2}$, Jaroslaw Hiczkiewicz ${ }^{3}$, Janusz Rzezniczak ${ }^{2}$ \\ ${ }^{1}$ Biology of Lipid Disorders Department, Chair of Biology and Environmental Sciences, \\ Poznan University of Medical Sciences, Poznan, Poland \\ ${ }^{2}$ Department of Cardiology, J. Strus Hospital, Poznan, Poland \\ ${ }^{3}$ Department of Cardiology, Hospital of Nowa Sol, Poland \\ ${ }^{4}$ Institute for Medical Immunology, Berlin-Brandenburg Center für Regenerative Therapies, \\ Core Unit Biomarker, Charité University Medicine Berlin, Germany \\ ${ }^{5}$ Zablab sp. $z$ o.o, Poznan, Poland \\ ${ }^{6}$ HaimaChek Inc., Santa Monica, CA, United States \\ ${ }^{7}$ Bayer AG, Biomarker Research, Berlin, Germany
}

\begin{abstract}
Background: Circulating endothelial cells (CEC) may be used to find new strategies for the early diagnosis of cardiovascular diseases. The major objective of the project is to broaden knowledge of CEC biology by determining their phenotypic characteristics. The additional aim is to clarify whether on the basis of these information it is possible to identify the origin of CEC release (from various cardiovascular compartments).

Methods: Circulating endothelial cells were collected from arterial blood prior to angiography, as well as from arterial and venous blood obtained after angiography/coronary angioplasty, from 18 patients with non-ST-segment elevation myocardial infarction (NSTEMI). CECs were quantified by flow cytometry and defined as Syto16 (dye) $)^{+}, C D 45 \mathrm{dim} / \mathrm{neg}, \mathrm{CD} 31^{+}$and $\mathrm{CD} 146^{+}$. The additional $C D 36^{+}$was establish as a marker of endothelial cells released from small vessels of the microcirculation.

Results: The total number of CECs increased significantly after the percutaneous transluminal coronary angioplasty (PTCA) in the arterial system. Number of CECs isolated at similar time points (after invasive procedure) did not differ significantly between arteries and veins, but the number of CD36 ${ }^{+}$ CECs after coronary angioplasty was significantly higher in the venous system, than in the arterial system. Conclusions: The number of $C D 36^{+}$in artery samples obtained after coronary angioplasty (PTCA) had tendency to be decreased (in comparison to the sample obtained before angiography). It was major difference between those who had PTCA performed vs. those who had not. (Cardiol J 2020; 27, 3: 295-302)
\end{abstract}

Key words: circulating endothelial cells, NSTEMI, diastolic dysfunction

Address for correspondence: Pawel Burchardt, PhD, MD, MSc, FESC, Biology of Lipid Disorders Department, Chair of Biology and Environmental Sciences, Poznan University of Medical Sciences; Department of Cardiology, J. Strus Hospital, ul. Szwajcarska 3, 61-285 Poznań, Poland, tel: +48 618739333, fax: + 48 618739333, e-mail: pab2@tlen.pl

Received: 19.01.2018 Accepted: 4.09.2018 


\section{Introduction}

The Holy Grail in cardiology is to 'predict the unpredictable'. This means, that $50 \%$ of all new cases of ischemic heart disease (IHD) occur as myocardial infarction or sudden cardiac death [1-7]. It is strange, but there are no clinical tools to predict and avoid these episodes. Neither can new cardio-vascular episodes be predicted among those who suffer from the established IHD [1].

Circulating endothelial cells (CEC) may be used to find early and new strategies for the diagnosis of cardiovascular diseases [8-13]. They are released into the blood due to reduced adhesion to the vessels basement membrane (VBM) as a result of mechanical injury, necrosis or apoptosis $[8,13$, 14]. Very little is known about the CEC phenotype, which may depend on their state of activation, way of release, vascular beds they originate from or the caliber of the vessel from which they are derived. The isolation and determination of CEC phenotype may allow, in combination with troponin assessment, for more sophisticated diagnosis of patients with acute coronary syndrome (ACS), as well as to distinguish from this group subjects with impaired coronary microcirculation. Additionally, it could be possible that based on the assessment of CEC phenotype, patients who are at risk for a new or another cardiovascular event would be identified.

Owing to the above, it was decided to focus on a cohort with a new onset of coronary artery disease in shape of ACS (with no history of cardiovascular events). The CECs level can be increased in all ACS patients, but the most pleasant objects for CEC studies are subjects with non-ST-segment elevation myocardial infarction (NSTEMI). The first reason is the wide variety of CEC sources (large epicardial CEC, microvascular CEC's) and the mechanisms leading to their release, i.e. mechanical injury and microvascular disturbances.

Secondly, the fact that according to European Society of Cardiology (ESC) guidelines more time is possible for clinical evaluation of NSTEMI patients before the decision to perform the diagnostic angiography to be done.

The purpose of the study is to:

- find differences in number of CECs between subjects who underwent percutaneous transluminal coronary angioplasty (PTCA) and those who did not;

- find quantitative differences between CECs depending on vascular beds that they are derived from (venous system vs. arterial system);

- establish the origin of isolated CECs depending on the compartment of arterial system of origin (artery vs. microcirculation);

- find differences in the amount of CECs isolated from the arterial system, depending on the way of their release before and after mechanical injury, (i.e. before and after PTCA).

\section{Methods}

\section{Patients and study design}

The study was carried out at the Department of Cardiology of J. Strus Hospital, Poznan, Poland between 2014 and 2016. Protocol was conducted according to guidelines stated in the Declaration of Helsinki and was approved by the local bioethics commission. All subjects were informed about the aim of the study and gave their written consent.

The study group consisted of patients suffering from ACS/NSTEMI/their first cardiovascular episode ever. The additional inclusion criteria was an impaired left ventricular (LV) diastolic function in echocardiography. Left diastolic dysfunction was defined when all echocardiographic features at admission were observed: $\mathrm{E} / \mathrm{A}<1$, e'/a' $<1$ from lateral wall, isovolumetric relaxation time $>0.1 \mathrm{~s}$, deceleration time of wave $\mathrm{E}$ was $>0.15 \mathrm{~s}$.

Patients were qualified for an acute coronary angiography (due to the ACS guidelines). Research material was arterial blood obtained before angiography (when the arterial sheet was fixed) and after coronary angiography/angioplasty (blood was collected from the arterial sheet before it was released after the invasive procedure). The last, venous blood collection was done from the ulnar vein. It was performed strictly $30 \mathrm{~min}$ after removing the arterial sheet after the angiography/angioplasty.

The exclusion criteria was lack of diastolic dysfunction in ACS/NSTEMI subjects, observed in screening echocardiography before diagnostic coronary angiography.

Coronary angiography was performed no later than $24 \mathrm{~h}$ after admission, in accordance with the ESC guidelines for management in NSTEMI.

The study was organized in three phases:

- selection of 18 patients with appropriate clinical characteristics;

- identification and quantification of CECs and microvascular CEC (mvCECs) using flow cytometry;

- statistical analysis of collected data. 


\section{CEC quantification and characterization by flow cytometry}

Circulating endothelial cells were detected by flow cytometry using a panel of monoclonal antibodies and nuclear staining Syto16. CECs were defined as $\mathrm{DNA}^{+}, \mathrm{CD} 45^{\mathrm{dim}}$, CD $31^{+}$and $\mathrm{CD} 146^{+}$. mvCECs were identified as CD $36^{+} \mathrm{CEC}$. In the staining procedure, whole blood samples $(500 \mu \mathrm{L})$ were incubated with FcR blocking Reagent (Miltenyi) for $15 \mathrm{~min}$ at $4^{\circ} \mathrm{C}$, and then with the respective antibody mixtures for $40 \mathrm{~min}$ at $4^{\circ} \mathrm{C}$. After red cell lysis with High-Yield Lyse (Life technologies) for $15 \mathrm{~min}$ at room temperature, samples were centrifuged and resuspended in FACS buffer (PBS + 0.5\% BSA + 0.5 mM EDTA + 0.05\% $\mathrm{NaN}_{3}$ ). Due to high variability associated with the detection of cell populations with low frequency, samples were stained and measured in triplicate. Acquisition was done using a LSR II Flow Cytometer (BD Biosciences), equipped with 488-, 633-, and 405-nm lasers. Flow cytometer setup and calibration were performed using CS\&T beads (BD Biosciences). For sample acquisition, mononuclear cells (PMNCs) were set as the stopping gate with a threshold of $5 \times 10^{5}$ PMNCs. Data was acquired using FACSDiva 6.0 Software (BD Biosciences) and data analysis was performed using Flowjo 10. Fluorescence compensation was performed by using $\mathrm{BD}^{\mathrm{TM}}$ CompBeads Set Anti-Mouse Ig. CECs and EPCs levels were first calculated as a percentage of PMNC. Absolute counts (cells $/ \mathrm{mL}$ ) were then determined by multiplying the CEC or EPC percentage of the PMNC by the absolute PMNC count obtained in separate tubes by using Flow-Count ${ }^{\mathrm{TM}}$ Fluorospheres (Beckmann Coulter).

\section{Laboratory tests}

Laboratory tests were performed using commercially available diagnostic kits. The serum creatinine concentration was determined by Jaffe's reaction using Roche Cobas C (Hitachi, Germany). Creatinine clearance and glomerular filtration rate were estimated using formulas from CockroftGault. Potassium and sodium concentrations were determined by potentiometry using the Cobas System 6000 (Roche Diagnostics, Germany). Assessments of peripheral blood counts were performed using Sysmex XT2000i, the US system. Concentration of urea, uric acid, total cholesterol, triglycerides and high-density lipoproteins were quantified using enzymatic colorimetric method (Cobas C Roche/Hitachi, Germany) with specific reagents. The concentration of low-density lipoproteins was calculated using the Friedewald formula.

\section{Statistical analysis}

After applying the Shapiro-Wilks test to determine a normal distribution of data, noncategorical data distributed normally was expressed as mean (SD) and data distributed non-normally was expressed as median (interquartile range [IQR]). The Student t-test was used for variables with normal distribution (for two independent and dependent variables). The Mann-Whitney U test (for two independent variables) and the Sign test as well as the Wilcoxon matched pairs test (for two dependent variables) were used for variables without normal distribution. Statistical significance was set at $\mathrm{p}<0.05$. The correlation between variables showing a normal distribution was evaluated with the Pearson coefficient, whereas the Spearman rang correlation coefficient was applied for variables with a non-normal distribution. All analyses were performed with STATISTICA 7.0 (Statsoft, USA) and SPSS-20 (IBM, USA).

\section{Results}

\section{Demographic data}

The study group consisted of 18 patients (14 males, 4 females) with a median age of $66.6(60-73)$ years with NSTEMI, and with additional echocardiographic features of LV diastolic dysfunction; moreover, this was their first episode of cardiovascular disease. The median body mass index of the study group was $24.7(22.6-28.7) \mathrm{kg} / \mathrm{m}^{2}$. Each patient received pharmacotherapy before index hospitalization. The clinical, biochemical and demographic characteristics are provided in Table 1.

Coronary artery angiography was performed in all patients and additional coronary angioplasty was performed in $14(77.8 \%)$ cases. Among the patients who underwent angioplasty, 5 (27.8\%) had stents placed in the left descending artery, $3(16.7 \%)$ in left circumflex artery and $1(5.5 \%)$ in left intermedia artery. One patient received stents to both the left circumflex and left intermedia artery. The right coronary artery had been stented in $4(22.2 \%)$ subjects.

During hospitalization patients received angiotensin converting enzyme inhibitors ( $75 \%$ of the subjects), angiotensin receptor blockers (10\% of the subjects), beta-blockers in (85\% of the subjects), statins (100\% of the subjects), diuretics (20\% of the subjects), and mineralocorticoid receptor antagonists (10\% of the subjects).

\section{Echocardiographic characteristics}

The echocardiographic parameters at hospital admission are provided in Table 2 . LV diastolic 
Table 1. Biochemical, clinical and demographic characteristics of patients at hospital admission.

\begin{tabular}{|c|c|c|c|}
\hline & Median & Percent & Interquartile range \\
\hline \multicolumn{4}{|l|}{ Laboratory parameter } \\
\hline $\mathrm{RBC}\left[10^{6} / \mu \mathrm{L}\right]$ & 4.4 & & $4.2-4.7$ \\
\hline $\mathrm{HGB}[\mathrm{g} / \mathrm{dl}]$ & 13.8 & & $12.6-14.7$ \\
\hline HCT [\%] & 39.8 & & $38.2-42.5$ \\
\hline WBC $\left[10^{3} / \mu \mathrm{L}\right]$ & 7.1 & & $6.2-8.5$ \\
\hline $\mathrm{K}^{+}[\mathrm{mmol} / \mathrm{L}]$ & 4.2 & & $3.8-4.47$ \\
\hline TSH [ulU/mL] & 1.1 & & $0.7-2.1$ \\
\hline $\mathrm{TC}[\mathrm{mmol} / \mathrm{L}]$ & 4.9 & & $4.5-5.8$ \\
\hline LDL [mmol/l] & 3.1 & & $2.2-4.1$ \\
\hline $\mathrm{HDL}[\mathrm{mmol} / \mathrm{L}]$ & 1.3 & & $1.1-1.4$ \\
\hline TG [mmol/L] & 1.6 & & $1.1-1.8$ \\
\hline CRP [mg/L] & 1.7 & & $1.1-5.5$ \\
\hline BNP [pg/mL] & 89.6 & & 49.3-221.5 \\
\hline Creatinine $[\mu \mathrm{mol} / \mathrm{L}]$ & 94 & & 79-105 \\
\hline ALAT [U/L] & 19 & & $15-29$ \\
\hline Tn max [ng/L] & 2645 & & $1910-4244$ \\
\hline \multicolumn{4}{|l|}{ Radiology parameter } \\
\hline Mean time of coronary angiography + PTCA [min] & 27.7 & & $17-34$ \\
\hline The balloon inflation time during angioplasty [s] & 25 & & $12.5-30$ \\
\hline \multicolumn{4}{|l|}{ Number of patients with performed PTCA $=14$} \\
\hline \multicolumn{4}{|l|}{ Average number of implanted stent per patient $=1.14$} \\
\hline \multicolumn{4}{|l|}{ Demographic data } \\
\hline Dysglicemia (IFG, IGT, diabetes) & & $65 \%$ & \\
\hline Dyslipidemia & & $55 \%$ & \\
\hline Hypertension & & $55 \%$ & \\
\hline Chronic kidney disease & & $20 \%$ & \\
\hline Heart failure & & $15 \%$ & \\
\hline
\end{tabular}

RBC - red blood count; HGB — hemoglobin; HCT — hematocrit; WBC - white blood count; $\mathrm{K}^{+}$- potassium; TSH - thyroid stimulating hormone; TC - total cholesterol; LDL - low density lipoprotein; HDL - high density lipoprotein; TG - triglycerides; CRP - C-reactive protein; BNP - B-type natriuretic protein; ALAT — alanine aminotransferase; Tn — troponin; PTCA — percutaneous transluminal coronary angioplasty; IFG — impaired fasting glucose; IGT — impaired glucose tolerance

dysfunction (inclusion criteria) at various levels of severity were present in all patients.

There was no significant improvement observed in diastolic function in echocardiography the following day, subsequent to angiography (or coronary artery angioplasty following ACS) (data not provided).

Characterization of CECs present before angiography in radial artery, and after coronary angiography/angioplasty in radial artery and brachial vein

Characteristics and levels of CECs obtained in the studied groups are shown in Table 3. The number of CECs after the angiography/angioplasty increased in the arterial system in the whole group.
In the venous system, although observed CEC cell numbers were highest, differences were not statistically significant. The number of $\mathrm{CD} 36^{+}$ (microvascular) cells after coronary angiography/ /angioplasty did not change significantly in the arterial system, but was significantly higher in vein, although the blood was collected during a similar period following an invasive cardiac procedure (30 min after the invasive procedure).

A comparison between subjects who had coronary angioplasty performed vs. those who did not (in artery before and after angiography and artery vs. vein after angioplasty) is provided in Table 4. The number of CEC, CEC per $10^{6} \mathrm{PMNC}$, the percentage of mvCEC on total CECs, measured before and after angiography in artery (as well as before 
Table 2. Echocardiographic characteristics (before coronary-angiography).

\begin{tabular}{lcc}
\hline Echocardiographic parameter & Median & Interquartile range \\
\hline Ejection fraction [\%] & 58.5 & $55-63$ \\
Right ventricular diastolic diameter [mm] & 27 & $23-31$ \\
Interventricular septum diastolic diameter [mm] & 12.5 & $12-15$ \\
Posterior wall diastolic diameter [mm] & 11.5 & $10-12$ \\
Left ventricular end-diastolic diameter [mm] & 45.5 & $44-48$ \\
Left atrium diameter [mm] & 34.5 & $34-38$ \\
Diameter of aorta (valve ring) [mm] & 19.5 & $19-23$ \\
Mitral flow rate, wave E [m/s] & 0.4 & $0.38-0.56$ \\
Mitral flow rate, wave A [m/s] & 0.6 & $0.52-0.67$ \\
E/a' & 9.5 & $7-11$ \\
Deceleration time of E wave mitral flow [ms] & 196.5 & $156-247$ \\
Isovolumetric relaxation time [ms] & 118.5 & $104-133$ \\
Lateral wall, flow rate, wave e' (tissue Doppler) [m/s] & 0.05 & $0.04-0.07$ \\
Lateral wall, flow rate, wave a' (tissue Doppler) [m/s] & 0.07 & $0.05-0.08$ \\
Acceleration time of right ventricular outflow [ms] & 121 & $104-133$ \\
\hline
\end{tabular}

Table 3. Characterization and quantification of circulating endothelial cells (CEC) in the radial artery before angiography, and after coronary angiography/angioplasty from the radial artery and brachial vein.

\begin{tabular}{lccc}
\hline Parameter & $\begin{array}{c}\text { Before angiography } \\
\text { (artery A1) }\end{array}$ & $\begin{array}{c}\text { After angiography } \\
\text { (artery A2) }\end{array}$ & $\begin{array}{c}\text { After angiography } \\
\text { (vein) }\end{array}$ \\
\hline $\mathrm{CEC} / \mathrm{mL}$ & $63.9(35.8-91.2)$ & $99.1(49.8-189.9)^{*}$ & $123.0(28.4-299.2)$ \\
$\mathrm{CEC}$ per $10^{6} \mathrm{PMNC}$ & $23.4(13.0-32.4)$ & $42.1(22.8-61.9)^{* *}$ & $58.5(9.8-95.3) \#$ \\
$\mathrm{mvCEC} / \mathrm{mL}$ & $28.9(13.3-47.8)$ & $26.2(9.8-43.0)$ & $51.2(16.4-107.8)$ \\
$\mathrm{mvCEC} \%$ of total CEC & $53.5(34.0-78.3)$ & $23.2(11.2-44.8)^{* * *}$ & $41.4(14.5-77.6) \$$ \\
\hline
\end{tabular}

Data are shown as median (interquartile range). Arterial $1 \mathrm{vs}$. arterial 2: ${ }^{*} \mathrm{p}<0.005,{ }^{* *} \mathrm{p}<0.05,{ }^{* *} \mathrm{p}<0.05 ;$ Arterial $1 \mathrm{vs}$. vein: \#p $<0.05$; Arterial 2 vs. vein: $\$ p<0.05$

Table 4. The comparison between subjects who had percutaneous transluminal coronary angioplasty (PTCA) performed vs those who did not have the operation performed.

\begin{tabular}{lcc}
\hline Parameter & $\begin{array}{c}\text { Patients without PTCA } \\
\text { before angiography }\end{array}$ & $\begin{array}{c}\text { Patients with PTCA performed } \\
\text { before angiography }\end{array}$ \\
\hline $\mathrm{CEC} / \mathrm{mL}$ & Artery A1 & Artery A1 \\
$\mathrm{CEC}$ per $10^{6}$ PMNC & $255.5(85.5-425.5)$ & $63.4(35.7-87.4)$ \\
$\mathrm{mvCEC} / \mathrm{mL}$ & $48.6(28.8-68.5)$ & $21.1(12.4-27.9)$ \\
$\mathrm{mvCEC} \%$ to CEC [\%] & $90.5(46.1-135)$ & $36.5(16.5-49.5)$ \\
$\mathrm{CEC} / \mathrm{mL}$ & $53.8(31.1-76.4)$ & $55.9(43.3-80)$ \\
$\mathrm{CEC} \mathrm{per} 10^{6}$ PMNC & Artery A2 & Artery A2 \\
$\mathrm{mvCEC} / \mathrm{mL}$ & $401.8(53.9-749.7)$ & $88.7(33.6-188.6)$ \\
$\mathrm{mvCEC} \%$ to CEC [\%] & $73.5(20.6-126.4)$ & $42(19.9-61)$ \\
$\mathrm{CEC} / \mathrm{mL}$ & $92.5(44.4-140.6)$ & $25(8.8-42.2)$ \\
$\mathrm{CEC} \mathrm{per} 10^{6}$ PMNC & $50.6(19.3-81.2)$ & $30.2(14.6-45)$ \\
$\mathrm{mvCEC} / \mathrm{mL}$ & Vein & Vein \\
$\mathrm{mvCEC} \%$ to CEC [\%] & $403(72.1-734)$ & $135.3(34.8-230.8)$ \\
\hline
\end{tabular}

Data are shown as median (interquartile range). $\mathrm{P}=$ not significant. $\mathrm{CEC} / \mathrm{ml}-\mathrm{CEC}$ per $\mathrm{mL}$ of peripheral blood; $\mathrm{PMNC}-\mathrm{CEC}$ per $10^{6}$ peripheral mononuclear blood cells, $\mathrm{mvCEC} / \mathrm{mL}-\mathrm{CD} 36^{+}$per $\mathrm{mL}$ of peripheral blood, \%mvCEC to CEC - the CD $36^{+}$to all CEC per $\mathrm{ml}$ of peripheral blood 
angiography in artery and after angiography in vein) did not change significantly between patients who underwent PTCA and those who didn't. Proper analysis of dependent samples in the mentioned groups were unable to perform due to small number of subjects (only group comparisons were done).

\section{Coronary angiography characteristics and its potential influence on CEC counts}

In 1 patient a myocardial bridge, which significantly narrowed the left descending artery, was found. He did not have PTCA performed. One patient was diagnosed with severe coronary atherosclerosis and was qualified for acute surgical coronary artery by-pass grafting procedure, so no PTCA performed. Another subject showed no significant coronary stenosis. In this case PTCA was also not performed, but from the clinical data it was possible to diagnose takotsubo cardiomyopathy. Angioplasty with implantation of 2 drug-eluting stents was performed in 2 patients. Long-lasting (58, 50, $40 \mathrm{~min})$ and very complicated PTCA took place in 3 patients. High values of myocardial necrosis-troponin released markers were observed in 6 patients $(25000,6580,5904,4244,3280,2645$ $\mathrm{ng} / \mathrm{mL}$ ). In 1 patient, no reflow phenomenon was observed after angioplasty. In another patient, additional severe aortic stenosis was observed but PTCA had not been performed.

\section{Circulating endothelial cells and clinical data}

Total CECs and CD36 ${ }^{+}$mvCEC were associated with individual clinical parameters. The ratio of $\mathrm{CD} 6^{+}$to all CEC per $\mathrm{ml}$ in arterial blood before angiography correlated with hemoglobin level $(\mathrm{r}=$ $=0.61$ ) and reversely correlate with mean platelet volume (MPV; $r=-0.78$ ). MPV was positively correlated with CEC count $(r=0.57) . \mathrm{CD}^{+} 6^{+}$in arterial blood before angiography correlated with high density lipoprotein $(r=0.6)$, and reversely with triglycerides $(r=0.6)$.

In arterial blood after coronary angiography/ /angioplasty: CEC count was correlated with sodium and creatinine concentration $(r=0.49$, $\mathrm{r}=0.52$, respectively), $\mathrm{CD} 36^{+}$with creatinine and white blood count $(r=0.49, r=-0.55$, respectively. In vein blood CEC count was reversely correlated with total cholesterol and low density lipoprotein $(\mathrm{r}=-0.63, \mathrm{r}=-0.063$, respectively). Venous $\mathrm{CD}^{+} 6^{+}$were positively correlated with platelets $(\mathrm{r}=0.49)$ and reversely correlated with MPV $(\mathrm{r}=-0.56)$, Total CECs and CD36 ${ }^{+}$mvCEC were also associated with various echocardiographic markers of LV relaxation dysfunction. However, due to weak correlation and lack of repeatability, these results were not provided.

\section{Discussion}

Circulating endothelial cells were identified and described for the first time in the 1970s. However, available literature is ambiguous in several key issues $[8,14]$. In the beginning, there was no clear definition of the term "circulating endothelial cells" [15]. Discrepancies in the definition of CECs were caused by a lack of the consensus on the nature of specific surface antigens, as well as what the size and shape of isolated particles/cells needed to be [8]. For these reasons, results obtained from individual authors significantly differed one from another [14].

After finding consensus on phenotypic characteristics specific to the CECs $\left(\mathrm{CD} 146^{+}, \mathrm{CD} 31^{+}\right.$, $\mathrm{CD} 36^{+}$), another source of variability between different studies was caused by the different technologies used for identification and quantification of these cells [8, 15-17]. Immunomagnetic methods allowed for isolation of cells on the basis of one specified surface antigen, therefore with less of specificity than compared to flow cytometry, which can use several different fluorescent-active antibodies. Moreover, most of the studies aimed only to quantify circulating cells rather than qualitatively analyze them [16-20].

The presence of CECs in the bloodstream is a physiological phenomenon resulting from impaired adhesive interactions between the basement membrane and endothelium $[8,14,15]$. The observed increase in the number of CECs is caused by a temporary impairment of the adhesive homeostasis by mechanical injury, apoptosis or necrosis [8, 14, 15]. Moreover, released CECs have different phenotypes depending on the activation properties, mode of their release (necrosis, apoptosis, mechanical damage) or their vascular origin. The most important factors which contribute to pathogenesis of CECs production are myocardial ischemia, inflammation, diabetes as well as thyroid or renal disturbances [21]. In the present study no significant CEC association with dysglicemia was observed, dyslipidemia with reduced LV ejection fraction or hypertension was also not observed. There were also no quantitative and phenotypic differences in CEC isolated from arterial and venous blood samples among patients with dysglicemia, dyslipidemia and reduced ejection fraction. Nevertheless, proper statistical analysis was not possible due to the small group and large heterogeneity of the cohort. 
Literature data shows that the number of CECs detected in the arterial system or venous system are similar, while there is no data concerning CEC phenotype differences between these compartments $[8,14,15]$. The goal herein, was to compare the number and type of cells present in the arterial system after angiography/ angioplasty, and $30 \mathrm{~min}$ after an invasive procedure from the venous system. It was found that the total number of CEC cells isolated at similar time points did not differ significantly between arterial and venous compartments. Little variation in CEC count was probably due to different time points of cell isolation (30 min delay between arterial and venous blood collection), rather than differences between these vascular compartments. A significantly higher number of cells with $\mathrm{CD} 36^{+}$ phenotype was observed in the venous system when it was compared to artery samples (before or after angiography/angioplasty). This was the only difference between arterial and venous systems in the context of isolated endothelial cells, that hadn't been described previously in the literature.

In the studied cohort, the total number of CECs detected in the blood before angiography was lower than the number of CECs obtained after angiography/angioplasty from both arterial and venous system. It may be speculated that this confirms results of other authors and possible cause of the phenomenon is increased CEC release after mechanical injury during angioplasty $[8,14$, 15]. There was no such observation in those who had not been treated with PTCA. The number of CECs was high at baseline and in the following time it was increasing, but not significantly. It may be speculated, that it could be explained rather by chronic myocardial ischemia which leads to low or moderate CECs increase, than other causes. However, absolute variability of the CECs number between patients who had vs. those who had not been treated with PTCA was not relevant.

This pilot study also aimed to find various sources of CEC. In subjects with clinically relevant ischemia (according to ESC ACS guidelines) accompanied by clinical probability of microcirculatory disturbances represented by diastolic dysfunction which were hoped to collect CEC originated from different vascular beds. It was found and distinguished that $\mathrm{CD} 36^{+}$from CD36-. There were no significant differences in the number of $\mathrm{CD}^{+} 6^{+}$in various blood collection time points. Interestingly, the number of $\mathrm{CD}^{+} 6^{+}$in artery samples obtained after coronary angioplasty (PTCA) had tended to be decreased (in comparison to samples obtained before angiography). The $\mathrm{CD} 36^{+}$in following blood samples tended to be increased in patients who had no angioplasty performed. There was major difference between those who had PTCA performed vs. those who had not. This phenomenon could not be properly explained. It is possible that it may have been a chance result. Secondly, it may only be speculated that angioplasty preserves blood supply to the myocardium and also improves homeostasis of microcirculation, which reduces the unpleasant effect of ischemia and diminishes CEC release. Patients without PTCA had their ischemia not reversed (complex epicardial and microcirculatory ischemia), and could explain the $\mathrm{CD}^{+} 6^{+}$increase.

The clinical application of this study is that isolation and determination of CEC phenotype is possible and that angioplasty of the epicardial coronary artery may influence the profile of released endothelial cells. Further studies on quantitative $\mathrm{CEC}$ properties are required, the purpose is to allow better distinction in angina subjects and those with impaired coronary microcirculation.

\section{Limitations of the study}

Due to a very small size of the group studied and the fact that statistical analysis cannot be performed for some parameters, results of the project should be interpreted as descriptive statistics. Another limitation was that the CEC results were not compared to other clinical groups of patients (i.e. healthy volunteers), which however was not a primary endpoint of the project's plan. The purpose of the study was confirmation of the notion that CEC count's variability depends on their vascular origin. It is worth noting that although the study group was small due to the highly selective inclusion criteria, its undoubted advantage is its homogeneity (according to various sources of CEC). The blood was obtained from patients with suspected coronary microvascular disturbances and hemodynamic decompensation due to concomitant changes in the epicardial artery during the course of NSTEMI. Such groups with various sources of CEC in average patients have, as of yet, not been described in the literature.

Finally, there are no known markers that can be used to distinguish CECs derived from coronary microcirculation from other microcirculation locations. That is why, they were excluded from patients studied having suspected peripheral artery disease of any kind. The phenotypic features that differentiate CEC from various micro-circulating beds are a separate subject of interest. 


\section{Conclusions}

The number of CECs after PTCA increased significantly in the arterial system. In the venous system, although the observed CEC cell numbers were the highest, differences were not statistically significant.

The number of cells with $\mathrm{CD} 36^{+}$phenotype after coronary angioplasty did not change significantly in the arterial system, but was significantly higher in vein, although the blood was collected during a similar period following an invasive cardiac procedure.

\section{Conflict of interest: None declared}

\section{References}

1. Montalescot G, Sechtem U, Achenbach S, et al. 2013 ESC guidelines on the management of stable coronary artery disease: the Task Force on the management of stable coronary artery disease of the European Society of Cardiology. Eur Heart J. 2013; 34(38): 2949-3003, doi:10.1093/eurheartj/eht296, indexed in Pubmed: 23996286.

2. Burchardt P, Goździcka-Józefiak A, Siminiak T. [IGF-1--a new risk factor for coronary atherosclerosis]. Kardiol Pol. 2006; 64(11): 1297-1302, indexed in Pubmed: 17165168.

3. Kassab GS, Rider CA, Tang NJ, et al. Morphometry of pig coronary arterial trees. Am J Physiol. 1993; 265(1 Pt 2): H350-H365, doi: 10.1152/ajpheart.1993.265.1.H350, indexed in Pubmed: 8342652 .

4. Bugiardini R, Pozzati A, Ottani F, et al. Vasotonic angina: a spectrum of ischemic syndromes involving functional abnormalities of the epicardial and microvascular coronary circulation. J Am Coll Cardiol. 1993; 22(2): 417-425, indexed in Pubmed: 8166784.

5. Crea F. Angina pectoris and normal coronary arteries: cardiac syndrome X. Heart. 2004; 90(4): 457-463, doi: 10.1136/ hrt.2003.020594.

6. Maseri A, Crea F, Kaski J, et al. Mechanisms of angina pectoris in syndrome X. J Am Coll Cardiol. 1991; 17(2): 499-506, doi: 10.1016/s0735-1097(10)80122-6.

7. Bugiardini R, Bairey Merz CN. Angina with "normal” coronary arteries: a changing philosophy. JAMA. 2005; 293(4): 477-484, doi: 10.1001/jama.293.4.477, indexed in Pubmed: 15671433.

8. Erdbruegger U, Haubitz M, Woywodt A. Circulating endothelial cells: a novel marker of endothelial damage. Clin Chim Acta. 2006; 373(1-2): 17-26, doi: 10.1016/j.cca.2006.05.016, indexed in Pubmed: 16836991.

9. Bouvier CA, Gaynor E, Cintron JR, et al. Circulating endothelium as an indication of vascular injury. Thromb Diath Haemorrh. 1970; 40: 163 .
10. Woywodt A, Blann AD, Kirsch T, et al. Isolation and enumeration of circulating endothelial cells by immunomagnetic isolation: proposal of a definition and a consensus protocol. J Thromb Haemost. 2006; 4(3): 671-677, doi: 10.1111/j.1538-7836.2006.01794.x, indexed in Pubmed: 16460450.

11. Hladovec J, Prerovský I, Stanĕk V, et al. Circulating endothelial cells in acute myocardial infarction and angina pectoris. Klin Wochenschr. 1978; 56(20): 1033-1036, indexed in Pubmed: 723184.

12. Goon PKY, Lip GYH, Boos CJ, et al. Circulating endothelial cells, endothelial progenitor cells, and endothelial microparticles in cancer. Neoplasia. 2006; 8(2): 79-88, doi: 10.1593/neo.05592, indexed in Pubmed: 16611400.

13. Mutin M, Canavy I, Blann A, et al. Direct evidence of endothelial injury in acute myocardial infarction and unstable angina by demonstration of circulating endothelial cells. Blood. 1999; 93(9): 2951-2958, indexed in Pubmed: 10216090.

14. Schmidt DE, Manca M, Hoefer IE. Circulating endothelial cells in coronary artery disease and acute coronary syndrome. Trends Cardiovasc Med. 2015; 25(7): 578-587, doi: 10.1016/j. tcm.2015.01.013, indexed in Pubmed: 25753180.

15. Burger D, Touyz RM. Cellular biomarkers of endothelial health: microparticles, endothelial progenitor cells, and circu-lating endothelial cells. J Am Soc Hypertens. 2012; 6(2): 85-99, doi: 10.1016/j.jash.2011.11.003, indexed in Pubmed: 22321962 .

16. Lanuti P, Santilli F, Marchisio M, et al. A novel flow cytometric approach to distinguish circulating endothelial cells from endothelial microparticles: relevance for the evaluation of endo-thelial dysfunction. J Immunol Methods. 2012; 380(1-2): 16-22, doi: 10.1016/j.jim.2012.03.007, indexed in Pubmed: 22484509.

17. Sinning JM, Losch J, Walenta K, et al. Circulating CD31+/Annexin $\mathrm{V}+$ microparticles correlate with cardiovascular outcomes. Eur Heart J. 2011; 32(16): 2034-2041, doi: 10.1093/eurheartj/ ehq478, indexed in Pubmed: 21186238.

18. Blann AD, Woywodt A, Bertolini F, et al. Circulating endothelial cells. Biomarker of vascular disease. Thromb Haemost. 2005; 93(2): 228-235, doi: 10.1160/TH04-09-0578, indexed in Pubmed: 15711737.

19. Sabatier F, Camoin-Jau L, Anfosso F, et al. Circulating endothelial cells, microparticles and progenitors: key players towards the definition of vascular competence. J Cell Mol Med. 2009; 13(3): 454-471, doi: 10.1111/j.1582-4934.2008.00639.x, indexed in Pubmed: 19379144.

20. Bertolini F, Shaked Y, Mancuso P, et al. The multifaceted circulating endothelial cell in cancer: towards marker and target identification. Nat Rev Cancer. 2006; 6(11): 835-845, doi: 10.1038/ /nrc1971, indexed in Pubmed: 17036040.

21. Solovey A, Lin Y, Browne P, et al. Circulating activated endothelial cells in sickle cell anemia. N Engl J Med. 1997; 337(22): 1584-1590, doi: 10.1056/NEJM199711273372203, indexed in Pubmed: 9371854. 